\title{
Early development in Kelletia kelletii (Forbes, 1850) (Gastropoda: Buccinidae), an Eastern Pacific gastropod with planktonic larvae
}

\section{Desarrollo temprano en Kelletia kelletii (Forbes, 1850) (Gastropoda: Buccinidae), un gasterópodo del Pacífico oriental con larvas planctónicas}

\author{
Jann E Vendetti \\ Malacology Department, Natural History Museum of Los Angeles County, 900 Exposition Blvd., Los Angeles, \\ California 90007, U.S.A. \\ E-mail:jvendett@nhm.org
}

\begin{abstract}
Kelletia kelletii (Forbes, 1850) is an intertidal to subtidal marine buccinid gastropod with a range from California, USA, to Baja California, Mexico. Many characteristics of its mating behavior, general life history, and larval biology are known, but details about its larval morphology and behavior are lacking. Here, aspects of its larval development and morphology during early ontogeny are chronicled, including larval velar form and function, asymmetrical development, particle ingestion, larval yolk reserves, and larval shell morphology. Snail oviposition behavior was observed in aquaria and egg capsules were dissected at different stages of development and examined under optical and scanning electron microscopy. Egg capsules had undeveloped eggs and/or embryos that were not ingested by K. kelletii larvae. Hatching time (natural excapsulation) varied between 37 to $55 \mathrm{~d}$ depending on water conditions, and endogenous yolk reserves were present in most veligers at capsule emergence. Pre-hatching veligers could swim in the plankton if excapsulated at $27 \mathrm{~d}$ and had symmetrical velar lobes but different sized cephalic tentacles. At 2.5 weeks in the plankton, both cephalic tentacles and velar lobes were asymmetrical, with those on the larvae's right larger than those on their left. Larval shells were brittle and poorly mineralized at excapsulation but fully mineralized with an apertural beak and proto-siphonal canal by 2.5 weeks in the plankton. Particle capture and transport through the velar lobes to the mouth was possible in pre-hatching veligers, but ingestion only occurred in emerged veligers when yolk stores were depleted. Chronicling early ontogeny and its sequence, as in this study, is essential to the understanding of larval development and its evolution in gastropods, and to comparative studies of larval biology in the Buccinidae.
\end{abstract}

Key words: larval biology, planktotrophic, neogastropod, California, Mexico.

RESUMEN. Kelletia kelletii (Forbes, 1850) es un gasterópodo bucínido marino de zonas intermareales y submareales con un intervalo de distribución desde California, EUA, hasta Baja California, México. Se conocen muchas de las características de su apareamiento, historia de vida general y biología larvaria, pero poco se sabe sobre los detalles de su morfología y comportamiento larvario. Aquí, se describe una crónica de los aspectos de su desarrollo y morfología larvaria durante la ontogenia temprana, como la forma y función del velo larvario, el desarrollo asimétrico, la ingestión de partículas, las reservas vitelinas larvarias y la morfología de la concha larvaria. El comportamiento de oviposición de los caracoles se observó en los acuarios, y las ovicápsulas en diferentes estadios de desarrollo fueron diseccionadas y examinadas mediante microscopía óptica y electrónica de barrido. Las ovicápsulas tenían huevos y/o embriones sin desarrollar que no fueron ingeridos por las larvas de K. kelletii. El tiempo de eclosión (descapsulación natural) varió entre 37 y 55 d dependiendo de las condiciones del agua, y la mayoría de las larvas velígeras tenían reservas vitelinas endógenas al emerger de la cápsula. Las velígeras no eclosionadas podían nadar en el plancton si se descapsulaban a los 27 d y tenían lóbulos velares simétricos, pero tentáculos cefálicos de diferentes tamaños. A las 2.5 semanas en el plancton, los tentáculos cefálicos y los lóbulos velares eran asimétricos, y los del lado derecho de las larvas eran más grandes que los del lado izquierdo. Las conchas larvarias estaban frágiles y pobremente mineralizadas en la descapsulación, pero completamente mineralizadas con un pico de apertura y canal proto-sifonal a las 2.5 semanas en el plancton. La captura de partículas y su transporte por los lóbulos velares a la boca fue posible en las velígeras pre-eclosión, pero la ingestión solo ocurrió en las velígeras emergidas cuando las reservas vitelinas se habían agotado. La crónica de la ontogenia temprana y su secuencia, como la presentada en este estudio, es esencial para comprender el desarrollo larvario y su evolución en gasterópodos, y para los estudios comparativos de biología larvaria en Buccinidae.

Palabras clave: biología larvaria, planctotrófico, neogastrópodo, California, México.

\section{INTRODUCTION}

The Kellet's whelk, Kelletia kelletii (Forbes, 1850), is a mostly subtidal buccinid gastropod endemic to the Eastern Pacific with a range that spans from Monterey Bay, California (USA), to Baja California Sur (Mexico) (McLean 1978, Herrlinger 1981). It is a conspicuous snail with a bright

\section{INTRODUCCIÓN}

El buccino de Kellet, Kelletia kelletii (Forbes, 1850), es un gasterópodo bucínido principalmente submareal endémico del Pacífico oriental con un intervalo de distribución que se extiende desde la bahía de Monterey, California (EUA), hasta Baja California Sur (México) (McLean 1978, 
yellow to orange body and fairly large, high-spired shell often covered in pink and green algae. This species is dioecious and its mating aggregations can include 200-300 individuals (Rosenthal 1970, Morris et al. 1980, Lonhart 2001). Its biogeography, life history, and many of its larval characteristics have been described by MacGinitie and MacGinitie (1949), Rosenthal (1970), Morris et al. (1980), Lonhart (2001), Zacherl et al. (2003, Zacherl (2005), Romero et al. (2012), Rodriguez (2017), and Wilson (2017). Herein, additional aspects of this species' larval biology are documented, including pre- and post-hatching endogenous yolk reserves, veliger velar lobe morphology, particle capture and ingestion, asymmetrical development, and larval shell morphology. Kelletia kelletii snails were observed in aquaria and egg capsules were selected, dissected, and examined at different stages of larval development to chronicle the characteristics listed above. Larval shells and veligers were also examined using scanning electron microscopy (SEM) at early to late veliger stages.

In the more than 700 species within the gastropod family Buccinidae, egg capsules are laid in clutches, masses, towers, or capsule strings on benthic substrates that sometimes include the shells of conspecifics (e.g., Solenosteira, Neptunea, and Kelletia) (Power and Keegan 2001). Depending on the species, egg capsules can house a single embryo to more than 1,000 (Thorson 1950). Intracapsular embryos develop into either non-planktonic larvae that crawl out of their capsule as metamorphosed juveniles (direct development) (Romero et al. 2004) or planktonic veliger larvae that swim out of their capsule, may or may not be obligated to feed, and then metamorphose into benthic adults (planktonic development) (Pearce and Thorson 1967). Developmental mode is not known to vary within buccinid species (Miloslavich and Penchaszadeh 1994, Tan and Morton 1998).

During intracapsular development, buccinid larvae derive nourishment from their own yolk, intracapsular albumen (protein-rich fluid that fills the capsule), and/or by ingesting eggs (oophagy), young embryos (nurse eggs), and/or sibling larvae (adelphophagy) within their capsule. Unlike most buccinids in the North Pacific, K. kelletii has a planktonic developmental mode (Rosenthal 1970, Pechenik 1979, Romero et al. 2012) wherein early development, including the trochophore, occurs inside an egg capsule without nutritive intracapsular eggs or embryos (Rosenthal 1970). At excapsulation, larvae emerge from capsules as feeding veligers (Buckland-Nicks et al. 2002).

With few exceptions, documentation of larval development, and particularly larval morphology, in buccinid gastropods is lacking, especially for taxa with planktonic larvae. Accounts of detailed larval characteristics across this diverse family could inform studies of larval development, larval ecology, and developmental evolution (Collin and Moran 2018). Thus, this study is intended to complement the work that has documented much of early ontogeny in $K$. kelletii
Herrlinger 1981). Es un caracol conspicuo con un cuerpo de color amarillo brillante a naranja y una concha bastante grande con espirales altos a menudo cubierta de algas rosadas y verdes. Esta especie es dioica y sus agregaciones de apareamiento pueden incluir entre 200 y 300 individuos (Rosenthal 1970, Morris et al. 1980, Lonhart 2001). Su biogeografía, historia de vida y muchas de sus características larvarias han sido descritas por MacGinitie y MacGinitie (1949), Rosenthal (1970), Morris et al. (1980), Lonhart (2001), Zacherl et al. (2003), Zacherl (2005), Romero et al. (2012), Rodríguez (2017) y Wilson (2017). Aquí, se documentan aspectos adicionales de la biología larvaria de esta especie, incluidas las reservas vitelinas endógenas antes y después de la eclosión, la morfología del lóbulo velar de la velígera, la captura y la ingestión de partículas, el desarrollo asimétrico y la morfología de la concha larvaria. Se observaron caracoles de $K$. kelletii en acuarios y se seleccionaron, diseccionaron y examinaron ovicápsulas en diferentes estadios de desarrollo larvario para describir las características mencionadas anteriormente. Las conchas larvarias y las velígeras también se examinaron mediante microscopía electrónica de barrido (MEB) en las etapas tempranas y tardías de la velígera.

En las más de 700 especies de la familia de los gasterópodos Buccinidae, las ovicápsulas se colocan en nidadas, masas, torres o cadenas de cápsulas sobre sustratos bentónicos que a veces incluyen las conchas de conespecíficos (e.g., Solenosteira, Neptunea y Kelletia) (Power y Keegan 2001). Dependiendo de la especie, las ovicápsulas pueden albergar desde un solo embrión hasta más de 1,000 (Thorson 1950). Los embriones intracapsulares se desarrollan como larvas no planctónicas que se arrastran hasta salir de su cápsula como juveniles metamorfoseados (desarrollo directo) (Romero et al. 2004) o como larvas planctónicas velígeras que nadan para salir de su cápsula, pueden estar o no obligadas a alimentarse y luego se metamorfosean en adultos bentónicos (desarrollo planctónico) (Pearce y Thorson 1967). No se tienen conocimientos de que el modo de desarrollo varié entre las especies de bucínidos (Miloslavich y Penchaszadeh 1994, Tan y Morton 1998).

Durante el desarrollo intracapsular, las larvas bucínidas se nutren de su propio vitelo, de la albúmina intracapsular (líquido rico en proteínas que llena la cápsula) y/o al ingerir huevos (oofagia), embriones jóvenes (huevos nodriza) y/o larvas hermanas (adelfofagia) dentro de su cápsula. A diferencia de la mayoría de los bucínidos en el Pacífico Norte, $K$. kelletii tiene un modo de desarrollo planctónico (Rosenthal 1970, Pechenik 1979, Romero et al. 2012) en el que el desarrollo temprano, incluido el trocóforo, ocurre dentro de una ovicápsula sin huevos o embriones intracapsulares nutritivos (Rosenthal 1970). En la descapsulación, las larvas emergen de las cápsulas como velígeras capaces de alimentarse (Buckland-Nicks et al. 2002).

Salvo algunas excepciones, se carece de documentación del desarrollo larvario, y en particular de la morfología 
and provide additional information for comparative studies of development within and across neogastropod clades.

\section{MATERIALS AND METHODS}

Voucher material including adult shells, preserved egg capsules, and mounted larval shells are deposited in the University of California (UC) Museum of Paleontology (UCMP) extant shell collections and wet collections.

\section{Kelletia kelletii specimen collection and maintenance}

Twelve adult specimens of $K$. kelletii were collected in California by hand using scuba from subtidal kelp forests near Monterey ( $n=7: 4$ females, 3 males) by T. Herrlinger (UC Berkeley) in January 2006 and Santa Barbara $(n=5$ : 2 females, 3 males) by S. Anderson (UC Santa Barbara) in June 2007.

Snails were maintained in the Valley Life Sciences Building on the UC Berkeley campus in a climate- and light-controlled room in a re-circulating and aerated 10 -gallon saltwater aquarium. Automatic overhead fluorescent lights were kept on a 12-h light/day-dark/night cycle. Water temperature remained at $13-16{ }^{\circ} \mathrm{C}$ and was measured every other day using a standard glass aquarium thermometer mounted to the inside of the aquarium. Salinity was measured weekly using a salinity refractometer and maintained at 34-35.

One third of the tank water was replaced with artificial or natural seawater once per month. Artificial seawater was made with Instant Ocean Sea Salt (Instant Ocean, Blacksburg, Virginia) to 34 salinity following the manufacturer's instructions. Natural, filtered seawater was acquired from Hopkins Marine Station, Pacific Grove, California. Adult snails were fed shrimp or bivalves every 3 to 4 weeks.

\section{Behavioral, capsule, and larval observations}

Mating and oviposition behavior was checked for at least $4 \mathrm{~d}$ per week and observed from January 2006 through October 2009. To chronicle the sequence of developmental changes in early ontogeny, 65 egg capsules were removed randomly from within the same clutches as well as from different clutches. Only egg capsules for which the date of oviposition was known were used for data collection.

Embryos and larvae were excapsulated by hand (with forceps and dissecting scissors) to allow different stages of development to be sampled, drawn, and photographed. A random sample of 5 egg capsules from different clutches was used to estimate egg/embryo number per capsule, and 15 capsules were measured from their base to their escape aperture to determine their typical dimensions. The onset of velar structures, organs, larval pigment, and larval shell mineralization were observed and recorded. After observations were made, the contents of capsules were sacrificed and preserved. larvaria, en gasterópodos bucínidos, especialmente para taxones con larvas planctónicas. Los trabajos que detallan las características larvarias en esta familia diversa podrían orientar los estudios sobre el desarrollo larvario, la ecología larvaria y la evolución del desarrollo (Collin y Moran 2018). Por lo tanto, este estudio pretende complementar el trabajo que ha documentado gran parte de la ontogenia temprana de $K$. kelletii y proporcionar información adicional para estudios comparativos del desarrollo dentro y entre clados de neogastrópodos.

\section{MATERIALES Y MÉTODOS}

El material de evidencia, que incluye conchas de adultos, ovicápsulas preservadas y conchas larvarias montadas, se encuentra depositado en las colecciones de conchas existentes y las colecciones húmedas del Museo de Paleontología (UCMP) de la Universidad de California (UC).

\section{Recolección y mantenimiento de las muestras de Kelletia kelletii}

Se recolectaron 12 especímenes adultos de K. kelletii en California. Los especímenes se obtuvieron manualmente por medio de buceo autónomo en los bosques de algas submareales cerca de Monterey ( $n=7: 4$ hembras, 3 machos) por T. Herrlinger (UC Berkeley) en enero de 2006 y Santa Bárbara ( $n=5: 2$ hembras, 3 machos) por S. Anderson (UC Santa Barbara) en junio de 2007.

Los caracoles se mantuvieron en el Edificio de Ciencias de la Vida del Valle en el campus de UC Berkeley en una habitación con clima y luz controlada en un acuario de agua salada de 10 galones con aireación y recirculación. Las luces fluorescentes automáticas sobre los acuarios se mantuvieron en un ciclo de $12 \mathrm{~h}$ de luz/día-oscuridad/noche. La temperatura del agua se mantuvo a $13-16^{\circ} \mathrm{C}$ y se midió cada $2 \mathrm{~d}$ utilizando un termómetro de vidrio estándar para acuarios montado en el interior del acuario. La salinidad se midió semanalmente utilizando un refractómetro de salinidad y se mantuvo a $34-35$.

Una tercera parte del agua del tanque se reemplazó con agua de mar artificial o natural una vez al mes. El agua de mar artificial se hizo con sal marina Instant Ocean (Instant Ocean, Blacksburg, Virginia) siguiendo las instrucciones del fabricante para alcanzar una salinidad de 34. El agua de mar natural y filtrada se adquirió de la Estación Marina Hopkins, Pacific Grove, California. Los caracoles adultos fueron alimentados con camarones o bivalvos cada 3 a 4 semanas.

\section{Observaciones del comportamiento, las cápsulas y las larvas}

Al menos 4 d a la semana se revisó si había comportamiento de apareamiento y oviposición, y este se observó desde enero de 2006 hasta octubre de 2009. Para hacer una 
Intracapsular behavior was observed through transparent capsules laid by one female collected from Santa Barbara. Capsules, eggs, larvae, and swimming veligers were observed under magnification with a Wild Heerbrugg M-series stereomicroscope. Digital photographs and digital videos were taken through this microscope using an optical coupler (Optem) and Coolpix (Nikon) 995 camera. The total number of eggs, embryos, and larvae examined was not counted but would be 32,500 if there were 500 eggs, embryos, or larvae in each of the 65 examined capsules.

\section{Larval feeding}

In 5 trials, at least 50 veliger larvae that naturally emerged from their capsules and at least 50 that were excapsulated by hand were transferred by glass pipettes to Syracuse watch glasses filled with micro-filtered seawater. To test for feeding ability, a solution of $100 \mathrm{~mL}$ seawater was mixed with approximately 1 teaspoon of red or blue nontoxic micro-particles (DayGlo fluorescent pigment; DayGlo Color Corporation, Cleveland, Ohio), which was introduced into the water via a pipette. Veligers were left undisturbed in this particle-rich seawater for $10 \mathrm{~min}$ before being checked for ingested particles, which would be visible in their gut through their translucent larval shell. If after 10 min none had particles visible within their gut, they were considered unable to feed; if particles were visible, they were considered planktotrophic.

\section{Larval shells: preparation for scanning electron microscopy}

Ontogenetic series of larval shells (of at least 3 specimens each) were prepared from 35 samples of both intracapsular and emerged veligers. Isolated larval shells were prepared for SEM analysis following a modified protocol of Pedersen and Page (2000): veligers were gently removed from beakers using a pipette, anaesthetized in $\mathrm{MgCl}_{2}$ for $2 \mathrm{~h}$, then bathed in a 3:10 (bleach: water) solution for up to $18 \mathrm{~h}$. Larval shells were rinsed with deionized water, allowed to dry on lint-free paper, then transferred by a fine sable brush into glass vials filled with acetone prior to mounting.

Cleaned larval shells were mounted with a sable brush onto conductive carbon adhesive pads on aluminum SEM stubs. Shells were repositioned when necessary using a paintbrush hair mounted on a wooden stirring stick. Mounted specimens were sputter coated with iridium on a Medo20 Sputter Coater to a thickness of $0.014 \mathrm{k} \AA$ (kiloangstrums) and visualized with a Philips XL-30 ESEM. Specimens were viewed at $10-15 \mathrm{kV}$ accelerating voltage and $15 \mathrm{~mm}$ working distance. Digital micrographs were taken using Philips XL-30 imaging software, and contrast and brightness were adjusted in Adobe Photoshop CS4. From these images, characteristics of larval shell ornamentation, microstructure, and shape were observed and recorded. Measurements were crónica de la secuencia de los cambios en el desarrollo en la ontogenia temprana, se extrajeron 65 ovicápsulas al azar de las mismas nidadas y de diferentes nidadas. Para la recopilación de datos, solo se utilizaron las ovicápsulas para las cuales se conocía la fecha de oviposición.

Los embriones y las larvas fueron descapsuladas a mano (con pinzas y tijeras de disección) para poder muestrear, dibujar y fotografiar las diferentes etapas de desarrollo. Se utilizó una muestra aleatoria de 5 ovicápsulas de diferentes nidadas para estimar el número de huevos/embriones por cápsula, y se midieron 15 cápsulas desde su base hasta su apertura de escape para determinar sus dimensiones típicas. Se observó y registró la aparición de estructuras velares, órganos, pigmento larvario y mineralización de la concha larvaria. Después de realizar las observaciones, el contenido de las cápsulas fue sacrificado y preservado.

El comportamiento intracapsular se observó a través de cápsulas transparentes puestas por una hembra recolectada en Santa Bárbara. Se observaron cápsulas, huevos, larvas y velígeras nadadoras bajo aumento con un estereomicroscopio Wild Heerbrugg serie M. Se tomaron fotografías digitales y videos digitales a través de este microscopio utilizando un acoplador óptico (Optem) y una cámara Coolpix (Nikon) 995. No se contó el número total de huevos, embriones y larvas examinados, pero sería 32,500 si hubieron 500 huevos, embriones o larvas en cada una de las 65 cápsulas examinadas.

\section{Alimentación de las larvas}

En 5 ensayos, al menos 50 larvas veliger que emergieron naturalmente de sus cápsulas y al menos 50 que fueron descapsuladas a mano fueron transferidas con pipetas de vidrio a vidrios de reloj Syracuse llenos de agua de mar microfiltrada. Para examinar la capacidad de alimentación, se mezcló una solución de $100 \mathrm{~mL}$ de agua de mar con aproximadamente 1 cucharadita de micropartículas no tóxicas rojas o azules (pigmento fluorescente DayGlo; DayGlo Color Corporation, Cleveland, Ohio), la cual se introdujo en el agua a través de una pipeta. Las velígeras permanecieron intactas en esta agua de mar rica en partículas durante 10 min antes de ser revisadas para identificar partículas ingeridas, las cuales serían visibles en sus intestinos a través de su concha larvaria traslúcida. Si después de 10 min ninguna tenía partículas visibles dentro de su intestino, se consideró que no podían alimentarse; si las partículas eran visibles, se consideraban planctotróficas.

\section{Conchas larvarias: preparación para la microscopía electrónica de barrido}

Se prepararon series ontogenéticas de conchas larvarias (de al menos 3 especímenes cada una) a partir de 35 muestras de velígeras tanto intracapsulares como emergidas. Se prepararon conchas larvarias aisladas para el análisis MEB 
made using ImageJ digitizing and imaging software (v.1.36b, National Institutes of Health; Image J 2004).

\section{Larvae: preparation for scanning electron microscopy}

Veliger larvae were relaxed in calcium-free seawater (Louise Page, pers. comm.), fixed in a $2 \%$ glutaraldehyde solution, rinsed in an osmium tetroxide and sodium cacodylate buffer, and dehydrated in a 7-step ethanol series from $35 \%$ to $99 \%$ ethanol. Fixed specimens were then critical point dried and visualized uncoated using a Hitachi TM-1000 SEM at an accelerating voltage between 15 and $20 \mathrm{kV}$.

\section{RESULTS}

\section{Shell size, mating, and oviposition}

Mean maximum length (ML) (from apex to siphonal canal tip) of shells collected in Santa Barbara snails $(n=5)$ was greater than that in Monterey-collected snails $(n=6)$ at $\mathrm{ML}=$ $92.5 \mathrm{~mm}, \mathrm{SD}=8.5 \mathrm{~mm} v s . \mathrm{ML}=85.2 \mathrm{~mm}, \mathrm{SD}=7.8 \mathrm{~mm}$. The same was true for mean maximum width (MW) (i.e., Santa Barbara-collected specimens: $\mathrm{MW}=51.6 \mathrm{~mm}, \mathrm{SD}=$ $2.9 \mathrm{~mm}$; Monterey-collected specimens: $\mathrm{MW}=44.3 \mathrm{~mm}$, $\mathrm{SD}=4.7 \mathrm{~mm}$ ). Males and females from Santa Barbara were not significantly different in shell length or width. Although the sample size is small, in Monterey-collected snails, the mean ML of shells was larger in females than in males: for females $(n=4) \mathrm{ML}=86.4 \mathrm{~mm}, \mathrm{SD}=8.9 \mathrm{~mm}, \mathrm{MW}=$ $45.6 \mathrm{~mm}, \mathrm{SD}=4.7 \mathrm{~mm}$; for males $(n=3) \mathrm{ML}=81.9 \mathrm{~mm}$, $\mathrm{SD}=5.0 \mathrm{~mm}, \mathrm{MW}=42.2 \mathrm{~mm}, \mathrm{SD}=3.6 \mathrm{~mm}$.

In aquaria, Santa Barbara-collected snails $(n=5)$ mated from January-February, and Monterey-collected snails $(n=6)$ mated from May-June. Females tended to lay egg capsules next to clutches already laid by other females on the glass sides of the aquarium, on rocks, and in one case, on the shell of another adult (Fig. 1a). Oviposition while mating was observed twice.

Sixteen instances of egg laying (oviposition) were recorded between May and October from 2006 to 2008: 3 in May, 6 in June, 3 in July, and 4 in August. The identity of the female that was ovipositing could be determined in 8 instances, and of these, the same Monterey-collected female oviposited from May-July, and 2 Santa Barbara-collected females oviposited from July-August.

\section{Egg capsules and intracapsular eggs and embryos}

Egg capsules were laid in benthic clutches of 3 to 82 capsules per clutch $(n=15$, mean $=25.1, \mathrm{SD}=23.3)$. In most capsules, the escape aperture and capsular plug were at the capsule top (Fig. 1b), although in one clutch the escape aperture was on the side of all capsules. Capsule walls were mostly translucent white to rarely transparent, and in the specimens examined here, rarely enclosed zero eggs or embryos. Egg/ siguiendo un protocolo modificado de Pedersen y Page (2000): las velígeras se retiraron suavemente de los vasos de precipitado con una pipeta, se anestesiaron en $\mathrm{MgCl}_{2}$ durante $2 \mathrm{~h}$ y posteriormente se bañaron en una solución 3:10 (cloro:agua) por hasta $18 \mathrm{~h}$. Las conchas larvarias se enjuagaron con agua desionizada, se dejaron secar sobre papel sin pelusa y se transfirieron con un pincel de marta fino a viales de vidrio que fueron llenados con acetona antes del montaje.

Las conchas larvarias limpias se montaron con un pincel de marta sobre cintas conductoras adhesivas de carbón sobre portamuestras de aluminio de MEB. Las conchas se reposicionaron cuando fue necesario utilizando un pelo de pincel montado en un agitador de madera. Los especímenes montados se recubrieron por pulverización catódica con iridio en un recubridor por pulverización Medo20 hasta un espesor de $0.014 \mathrm{k} \AA$ (kilo-angstroms) y se visualizaron con un microscopio electrónico de barrido ambiental Philips XL-30 ESEM. Las muestras se observaron con un voltaje de aceleración de 10 a $15 \mathrm{kV}$ y una distancia de trabajo de $15 \mathrm{~mm}$. Se tomaron micrografías digitales utilizando el software de imágenes Philips XL-30, y el contraste y el brillo se ajustaron en Adobe Photoshop CS4. Estas imágenes se usaron para observar y registrar las características de la ornamentación, microestructura y forma de la concha larvaria. Las mediciones se realizaron utilizando el software de digitalización y tratamiento de imágenes ImageJ (v.1.36b, National Institutes of Health; Image J 2004).

\section{Larvas: preparación para la microscopía electrónica de barrido}

Las larvas velígeras se relajaron en agua de mar sin calcio (Louise Page, com. pers.), se fijaron en una solución de glutaraldehído al $2 \%$, se enjuagaron en un tampón de tetróxido de osmio y cacodilato de sodio y se deshidrataron en una serie de etanol de 7 pasos desde etanol al 35\% hasta 99\%. Posteriormente, los especímenes fijados se secaron en el punto crítico y se visualizaron sin recubrir usando un MEB Hitachi TM-1000 a un voltaje de aceleración entre 15 y 20 kV.

\section{Resultados}

\section{Tamaño de la concha, apareamiento y oviposición}

La longitud máxima (LM) media (desde el ápice hasta la punta del canal sifonal) de las conchas recolectadas de los caracoles de Santa Bárbara $(n=5)$ fue mayor que la de los caracoles recolectados en Monterey $(n=6)$ con una $\mathrm{LM}=92.5 \mathrm{~mm}$, desviación estándar $(\mathrm{DE})=8.5 \mathrm{~mm} v s$. $\mathrm{LM}=85.2 \mathrm{~mm}, \mathrm{DE}=7.8 \mathrm{~mm}$. Se observó lo mismo para el ancho máximo (AM) medio (i.e., muestras recolectadas en Santa Bárbara: $\mathrm{AM}=51.6 \mathrm{~mm}, \mathrm{DE}=2.9 \mathrm{~mm}$; muestras recolectadas en Monterey: $\mathrm{AM}=44.3 \mathrm{~mm}, \mathrm{DE}=4.7 \mathrm{~mm}$ ). Los machos y las hembras de Santa Bárbara no fueron 
embryo number varied between 477 and 966 per capsule $(n=$ 5 , mean $=887.4, \mathrm{SD}=183.8)$. Capsules averaged $8.09 \mathrm{~mm}$ in height from their base to escape aperture $(n=15, \mathrm{SD}=1.1)$ and $6.60 \mathrm{~mm}$ in maximum width $(n=15, \mathrm{SD}=0.34)$. Almost all were lingulate, slightly convex/concave, and without sutures. Capsule thickness was not measured.

\section{Larval development}

Development in $13-16{ }^{\circ} \mathrm{C}$ seawater was observed from uncleaved fertilized eggs, to trochophores, to excapsulated swimming veligers. Eggs and trochophores were bright yellow to orange. Black pigment developed on veligers prior to their emergence and this color change made the translucent capsules appear gray (Fig. 1b). The onset and sequence of larval traits from the day of oviposition are summarized below and in Figure 2.

At the day of oviposition, zygotes were observed as uncleaved eggs (Fig. 3a). Cell differentiation into 4-8 cells was evident at 2-3 d (Fig. 2, 3b). The post-gastrula to trochophore developed by 9-11 d (Fig. 3c). At the late trochophore stage, embryos slowly rotated inside capsules. At 18-19 d, eye spots were present and some larvae had black pigment. Early veligers were evident at $19-21 \mathrm{~d}$ and they were closely packed and had a larval shell, velum, and red-colored larval kidney. After $21 \mathrm{~d}$, veligers showed ciliary movement and black pigment had become a prominent ring around the shell margin. At 23-25 d after oviposition, veligers actively swam inside the capsule with small velar lobes. At 27 d, if excapsulated, veligers were phototaxic and competent swimmers with 2 endogenous yolk balls in their shell (Fig. 3d). By $30 \mathrm{~d}$ veligers had well-developed black pigment. Their operculum was obvious by $32 \mathrm{~d}$. At $33 \mathrm{~d}$, cephalic tentacles were present and veligers released a grainy exudate. By 36-37 d, yolk reserved were small to absent, larval shells had holes and were not fully mineralized (Fig. 3e, f), and many veligers were nearly breaking through the capsular plug. If excapsulated at this time, veligers did not ingest micro-particles. At $38-55 \mathrm{~d}$, veligers emerged from their capsules as phototaxic planktonic veligers, some with small endogenous yolk reserves in their shells, some with holes in their shells, and all with black pigment on their foot. By day 45 swimming veligers had asymmetrical cephalic tentacles. At 49 d, there were no endogenous yolk reserves within the shells of most veligers. At 53-54 d, velar lobes were noticeably asymmetrical. At $69 \mathrm{~d}$, veligers could ingest micro-particles by trapping them in their velar lobes and bringing them toward the mouth. At $82 \mathrm{~d}$ after egg capsule deposition, few swimming veligers were alive without a food source.

\section{Velar form and function}

Feeding experiments indicate that opposed band cilia (the prototroch and metatroch) of velar lobes of $K$. kelletii significativamente diferentes en cuanto al largo o el ancho de la concha. Aunque el tamaño de la muestra es pequeño, en los caracoles recolectados en Monterey, la media de LM de las conchas fue mayor en las hembras que en los machos: para las hembras $(n=4) \mathrm{LM}=86.4 \mathrm{~mm}, \mathrm{DE}=8.9 \mathrm{~mm}$, $\mathrm{AM}=45.6 \mathrm{~mm}, \mathrm{DE}=4.7 \mathrm{~mm}$; para los machos $(n=3) \mathrm{LM}=$ $81.9 \mathrm{~mm}, \mathrm{DE}=5.0 \mathrm{~mm}, \mathrm{AM}=42.2 \mathrm{~mm}, \mathrm{DE}=3.6 \mathrm{~mm}$.

En los acuarios, los caracoles recolectados en Santa Bárbara $(n=5)$ se aparearon de enero a febrero, y los caracoles recolectados en Monterey $(n=6)$ se aparearon de mayo a junio. Las hembras tendieron a depositar las ovicápsulas junto a las nidadas que habían depositado otras hembras sobre los vidrios a los lados del acuario, sobre las rocas y, en un caso, sobre la concha de otro adulto (Fig. 1a). La oviposición durante el apareamiento se observó 2 veces.

Se registraron 16 casos de puesta de huevos (oviposición) entre mayo y octubre de 2006 a 2008: 3 en mayo, 6 en junio, 3 en julio y 4 en agosto. La identidad de la hembra que estaba ovipositando se pudo determinar en 8 casos; de estos, la misma hembra recolectada en Monterey ovipositó de mayo a julio, y 2 hembras recolectadas en Santa Bárbara ovipositaron de julio a agosto.

\section{Ovicápsulas y huevos y embriones intracapsulares}

Las ovicápsulas fueron depositadas en nidadas bentónicas, con 3 a 82 cápsulas por nidada $(n=15$, media $=25.1$, $\mathrm{DE}=23.3$ ). En la mayoría de las cápsulas, la apertura de escape y el tapón capsular estaban en la parte superior de la cápsula (Fig. 1b), aunque en una nidada la apertura de escape estaba en el lateral de todas las cápsulas. Las paredes de las cápsulas eran, en su mayoría, blancas translúcidas y pocas veces transparentes y, en los especímenes examinados aquí, rara vez encapsulaban cero huevos o embriones. El número de huevos/embriones varió entre 477 y 966 por cápsula $(n=5$, media $=887.4, \mathrm{DE}=183.8)$. Las cápsulas tenían una altura promedio de $8.09 \mathrm{~mm}$ desde su base hasta la apertura de escape $(n=15, \mathrm{DE}=1.1)$ y un ancho máximo promedio de $6.60 \mathrm{~mm}(n=15, \mathrm{SD}=0.34)$. Casi todas eran linguladas, ligeramente convexas/cóncavas y sin suturas. No se midió el grosor de la cápsula.

\section{Desarrollo larvario}

Se observó el desarrollo en agua de mar a $13-16^{\circ} \mathrm{C}$, desde huevos fertilizados no divididos hasta trocóforos y velígeras nadadoras descapsuladas. Los huevos y los trocóforos eran de color amarillo brillante a naranja. El pigmento negro se desarrolló en las velígeras antes de que emergieran y este cambio de color hizo que las cápsulas translúcidas parecieran grises (Fig. 1b). El inicio y la secuencia de los rasgos larvarios desde el día de la oviposición se resumen a continuación y en la Figura 2.

En el día de la oviposición, se observaron cigotos como huevos no divididos (Fig. 3a). La diferenciación celular en 

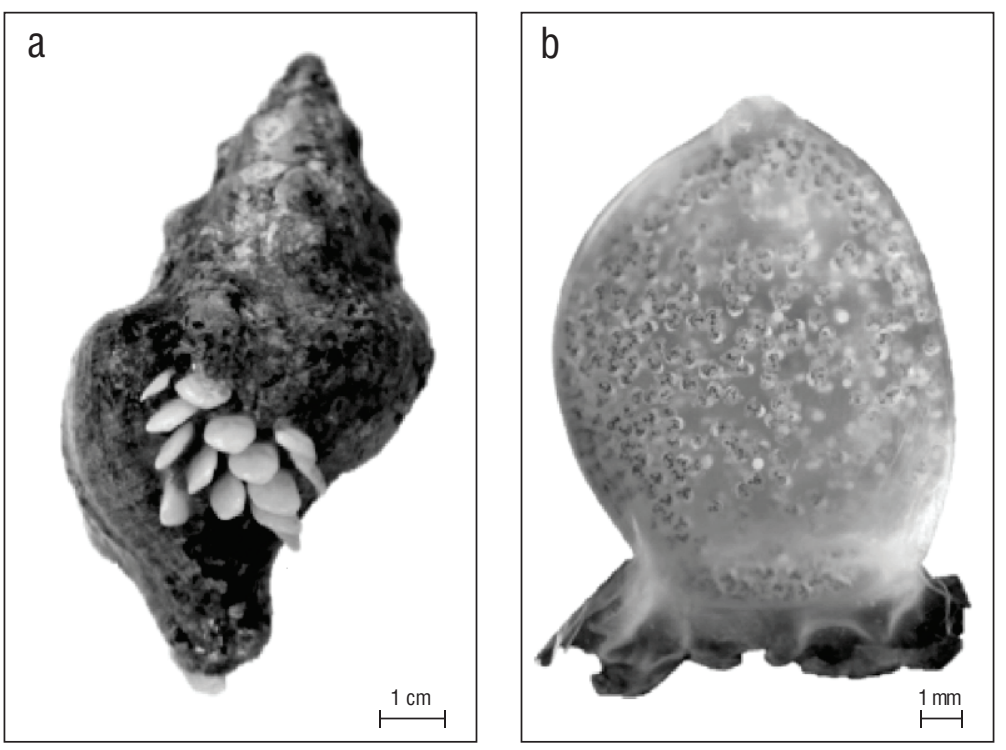

Figure 1. Kelletia kelletii egg capsules. (a) Living K. kelletii with egg capsules oviposited on its shell. (b) Single egg capsule with larvae nearly ready to emerge; capsule's escape aperture is at the top.

Figura 1. Ovicápsulas de Kelletia kelletii. (a) K. kelletii vivo con ovicápsulas ovipositadas en su concha. (b) Cápsula de un solo huevo con larvas casi listas para emerger; la apertura de escape de la cápsula está en la parte superior.

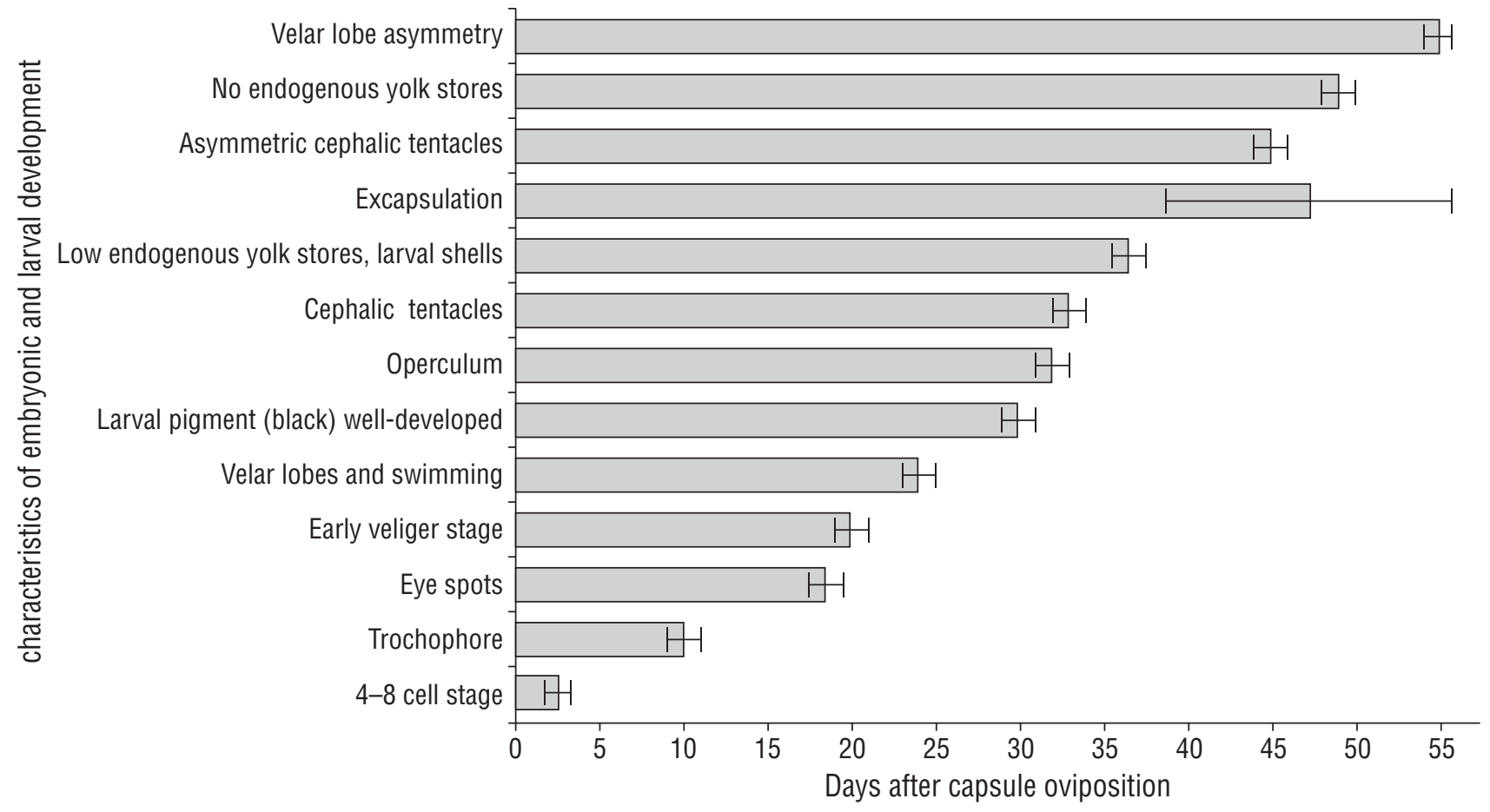

Figure 2. Characteristics of embryonic and larval development in Kelletia kelletii in aquaria at $13-16^{\circ} \mathrm{C}$ beginning at oviposition (day 0 ). Error bars indicate mean \pm SD. Graph design inspired by Horwitz et al. 2017.

Figura 2. Características del desarrollo embrionario y larvario en Kelletia kelletii en acuarios a $13-16{ }^{\circ} \mathrm{C}$ a partir de la oviposición (día 0). Las barras de error indican la media \pm desviación estándar. Diseño gráfico inspirado en Horwitz et al. 2017. 

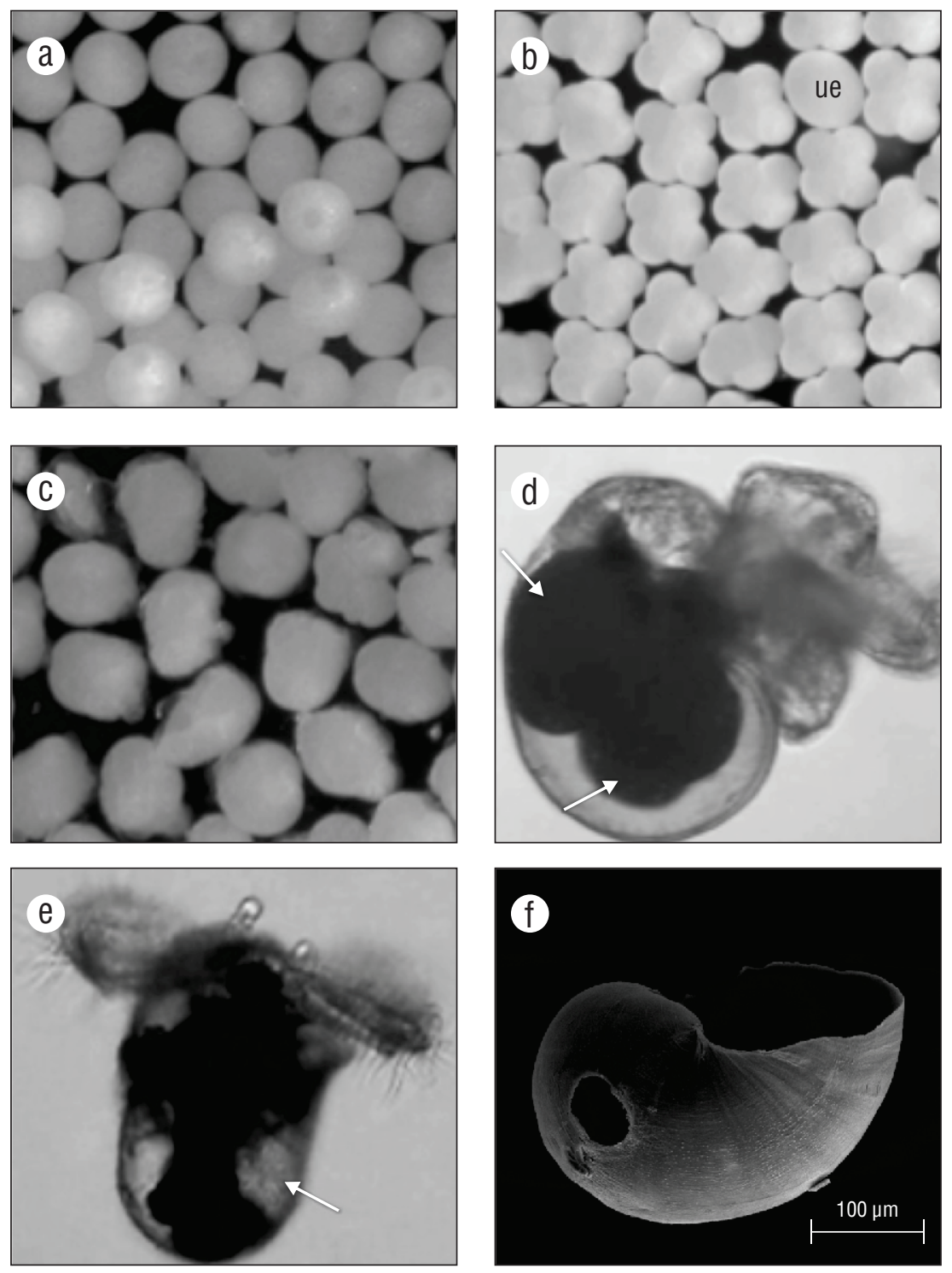

Figure 3. Kelletia kelletii embryonic and larval stages. (a) Zygote; (b) 4-8 cell stage (ue: egg or undeveloped embryo); (c) post-gastrula to trochophore stage; (d) encapsulated veliger (pre-hatching), with arrows indicating large endogenous yolk reserves; (e) excapsulated veliger (post-hatching), with arrow indicating low endogenous yolk reserve; and (f) SEM micrograph of late pre-hatchling larval shell with brittle edges and hole due to poor mineralization.

Figura 3. Etapas embrionarias y larvarias de Kelletia kelletii. (a) cigoto; (b) estadio de 4-8 células (ue: huevo o embrión no desarrollado); (c) etapa postgástrula a trocóforo; (d) velígera encapsulada (pre-eclosión), con flechas que indican grandes reservas vitelinas endógenas; (e) velígera descapsulada (post-eclosión), con una flecha que indica la baja reserva vitelina endógena; y (f) micrografía mediante microscopía electrónica de barrido de la concha larvaria de pre-cria tardía con bordes quebradizos y un agujero debido a una mineralización deficiente.

are capable of particle capture and transport in addition to swimming. Before veligers naturally hatched from their capsules, they used the velum to swim within the albumen of the capsule. All veligers that could swim outside of the capsule whether naturally hatched or excapsulated by hand were phototaxic. Before and after natural excapsulation, while yolk reserves were present, veligers could pull micro-particles from the water into the food groove of either velar lobe, but the particles would not be ingested; they would travel toward the mouth (Fig. 4) and be expelled down the foot.
4-8 células fue evidente a los $2-3 \mathrm{~d}$ (Fig. 2, 3b). La postgástrula se desarrolló a trocófora entre los 9 y 11 d (Fig. 3c). En la etapa tardía de la trocófora, los embriones rotaban lentamente dentro de las cápsulas. A los 18-19 d, había manchas oculares y algunas larvas tenían pigmento negro. A los 19-21 d, la presencia de velígeras tempranas era evidente; estas estaban muy compactas y tenían una concha larvaria, un velo y un riñón larvario de color rojo. Después de $21 \mathrm{~d}$, las velígeras mostraban movimiento ciliar y el pigmento negro se había convertido en un anillo prominente 


\section{Asymmetrical development}

Prior to hatching, veliger velar lobes were of equal size but cephalic tentacles were not (Fig. 5a, b). In all observed veligers, the right tentacle was longer than its counterpart on the left. By at least $50 \mathrm{~d}$ old or 2.5 weeks in the plankton, depending on the date of excapsulation, velar lobes were also asymmetrical, with the larva's right lobe larger than its left (Fig. 5c).

\section{Undeveloped eggs, yolk reserves, and particle ingestion}

Undeveloped or developmentally arrested embryos comprised $2.5 \%-38 \%$ of intracapsular individuals. In all dissected capsules $(n=65)$ there was a proportion of eggs/embryos that did not match the developmental stage of the majority of individuals in the capsule. This was true as early as the 4-cell stage as well as at veliger excapsulation. Undeveloped eggs were neither damaged nor were they in any stage of disintegration in all capsules examined, indicating that they had not been damaged or eaten by developing larvae. Observations through transparent capsules confirmed that trochophore and veliger larvae did not eat or disturb undeveloped eggs and embryos as they would have if these were nutritive material such as nurse eggs. Intracapsular embryos and veligers were fed by endogenous egg stores and possibly ingested protein-rich albumen.

Prior to hatching, bright yellow endogenous yolk reserves filled most of the veliger larval shell (Fig. 3d). Yolk was distributed into 1 or 2 ball-shaped reserves visible through the translucent shell. If veligers were excapsulated while yolk reserves were abundant, they would not ingest micro-particles. If micro-particles were introduced to an excapsulated pre-hatchling veliger, the pigment particles would get caught in the velar lobes, settle in the food groove, and move toward the mouth where they would be expelled down the ciliated foot. Veligers did not ingest particles unless their yolk stores were depleted (e.g., in some cases at $18 \mathrm{~d}$ in the plankton). Ingestion of micro-particles was confirmed by the bright color of the DayGlo micro-particles visible within the gut.

\section{Larval shell morphology}

Shells of late pre-hatching intracapsular veligers were thin, brittle, and often had holes indicating poor shell mineralization (Fig. 3f). Shells of emergent veligers were similar to those of pre-hatchlings but were slightly more mineralized and not as brittle. Between day 13 and 15 as a veliger, larval shells were fully mineralized and growth lines were evident (Fig. 6a, b, c). Late stage veligers at or near $20 \mathrm{~d}$ as excapsulated swimming veligers had a larval shell apertural beak and the beginnings of a siphonal canal (proto-siphonal canal) (Fig. 5c, 6c). In shells of veligers of different ages, beadlike micro-protuberances were evident up to the apertural margin (Fig. 6d, e, f). The maximum diameter of $K$. kelletii alrededor del margen de la concha. A los 23-25 d después de la oviposición, las velígeras nadaban activamente dentro de la cápsula con pequeños lóbulos velares. A los 27 d, las velígeras, si eran descapsuladas, eran fototáxicas, nadaban hábilmente y tenían 2 esferas vitelinas endógenas en su concha (Fig. 3d). A los 30 d, las velígeras tenían un pigmento negro bien desarrollado. Su opérculo era obvio a los 32 d. A los 33 d, los tentáculos cefálicos estaban presentes y las velígeras liberaban un exudado granulado. A los 36-37 d, la reserva vitelina era pequeña o ausente, las cochas larvarias tenían agujeros y no estaban completamente mineralizadas (Fig. 3e, f), y muchas velígeras casi rompían el tapón capsular. Si eran descapsuladas en este momento, las velígeras no ingerían micropartículas. A los 38-55 d, las velígeras emergieron de sus cápsulas como velígeras planctónicas fototáxicas, algunas con pequeñas reservas vitelinas endógenas en sus conchas, algunas con agujeros en sus conchas y todas con pigmento negro en el pie. A los $45 \mathrm{~d}$, las velígeras nadadoras tenían tentáculos cefálicos asimétricos. A los 49 d, no había reservas vitelinas endógenas dentro de las conchas de la mayoría de las velígeras. A los 53-54 d, los lóbulos velares eran notablemente asimétricos. A los $69 \mathrm{~d}$, las velígeras podían ingerir micropartículas atrapándolas en sus lóbulos velares y llevándolas hacia la boca. A los $82 \mathrm{~d}$ después de la puesta de la ovicápsula, pocas velígeras nadadoras estaban vivas sin una fuente de alimento.

\section{Forma y función del velo}

Los experimentos de alimentación indican que los cilios de bandas opuestas (el prototroca y metatroca) de los lóbulos velares de K. kelletii son capaces de capturar y transportar partículas además de ser natatorias. Antes de que las velígeras eclosionaran naturalmente de sus cápsulas, usaban el velo para nadar dentro de la albúmina de la cápsula. Todas las velígeras que podían nadar fuera de la cápsula, tanto las que eclosionaron de forma natural como las que fueron descapsuladas a mano, eran fototáxicas. Antes y después de la descapsulación natural, mientras las reservas vitelinas estuvieran presentes, las velígeras podían extraer micropartículas del agua hacia el surco alimentario de cualquiera de los lóbulos velares, pero las partículas no eran ingeridas; viajaban hacia la boca (Fig. 4) y eran expulsadas por el pie.

\section{Desarrollo asimétrico}

Antes de la eclosión, los lóbulos velares de las velígeras eran del mismo tamaño, pero los tentáculos cefálicos no (Fig. 5a, b). En todas las velígeras observadas, el tentáculo derecho era más largo que su contraparte izquierda. A por lo menos $50 \mathrm{~d}$ de edad o a las 2.5 semanas en el plancton, dependiendo de la fecha de descapsulación, los lóbulos velares también eran asimétricos, con el lóbulo derecho de la larva más grande que el izquierdo (Fig. 5c). 
larval shells was $0.407 \mathrm{~mm}(n=10)$ in apical view. However, overall size varied within capsules and cohorts were not always at the same stage of shell development and calcification, especially as pre-hatchlings and early-stage emerged veligers.

\section{Discussion}

Many observations of $K$. kelletii reported here are consistent with those documented elsewhere (e.g., by MacGinitie and MacGinitie 1949, Rosenthal 1970, Morris et al. 1980, Zacherl et al. 2003, Zacherl 2005, Romero et al. 2012, Rodriguez 2017, Wilson 2017). For example, larvae are nonfeeding within their egg capsules, excapsulated larvae swim and feed in the plankton as veligers, and excapsulated larvae are phototaxic. Notably, several observations of larval morphology and development are reported here for the first time: all examined egg capsules included developmentally arrested or non-fertilized eggs; after emergence from their capsule,

\section{Huevos sin desarrollar, reservas vitelinas e ingestión de partículas}

Los embriones no desarrollados o detenidos en el desarrollo correspondieron al $2.5 \%-38 \%$ de los individuos intracapsulares. En todas las cápsulas diseccionadas $(n=65)$ hubo una proporción de óvulos/embriones que no coincidió con el estadio de desarrollo de la mayoría de los individuos en la cápsula. Esto ocurrió desde etapas tan tempranas como la etapa de 4 células, así como en la descapsulación de velígeras. Los huevos no desarrollados no estaban dañados ni presentaron ninguna etapa de desintegración en todas las cápsulas examinadas, lo que indica que no habían sido dañados ni comidos por las larvas en desarrollo. Las observaciones a través las de cápsulas transparentes confirmaron que las larvas trocóforas y velígeras no comían ni perturbaban los huevos y embriones no desarrollados como lo hubieran hecho si estos tuvieran material nutritivo, como los huevos nodriza. Los embriones intracapsulares y las velígeras fueron
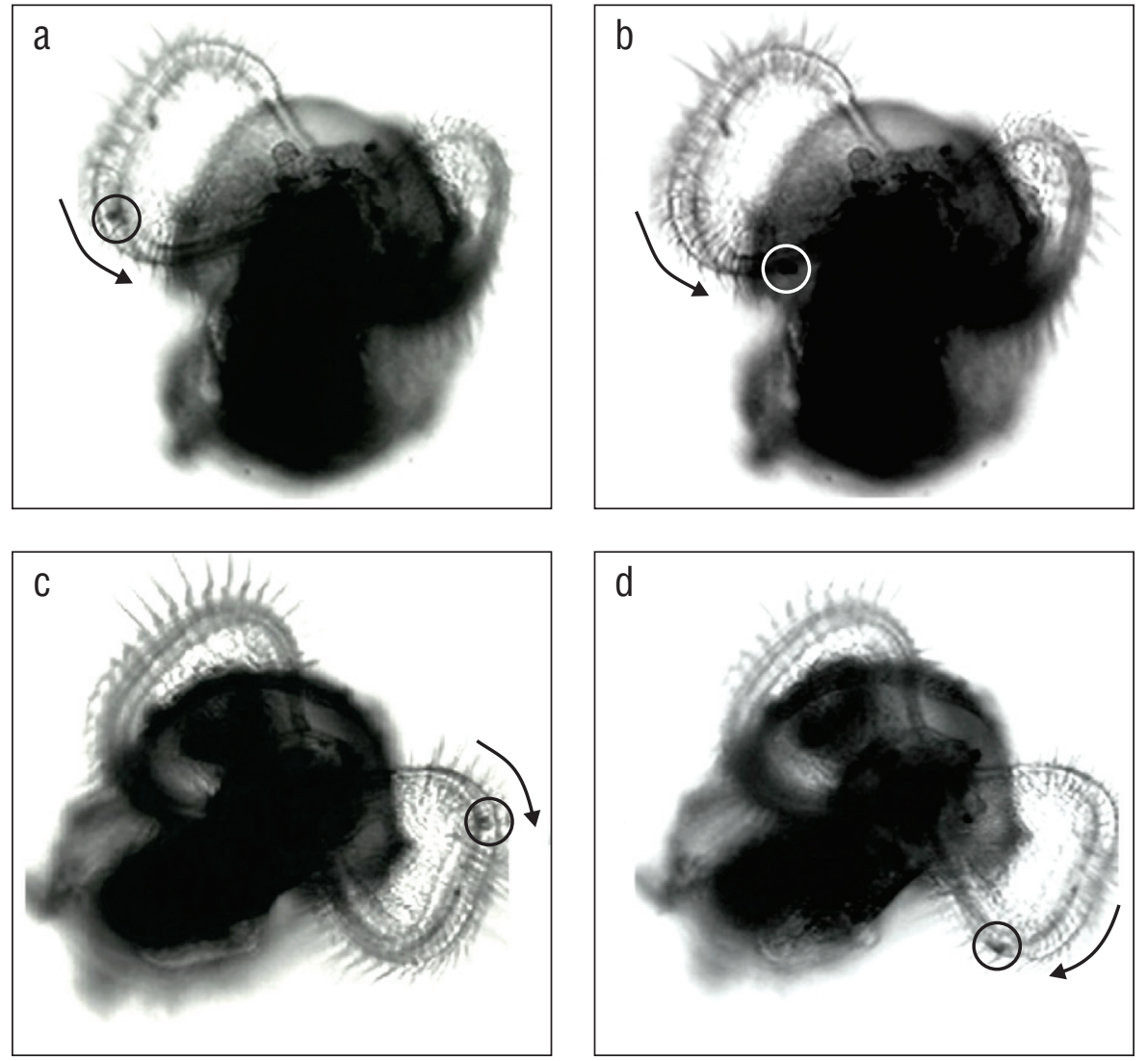

Figure 4. Particle capture and transport through the velar food groove in Kelletia kelletii veligers. Images were isolated from digital videos. Arrows indicate direction of particle movement. (a, b) Particle capture and counterclockwise movement in the right velar lobe of a post-hatched veliger. $(\mathbf{c}, \mathbf{d})$ Particle capture and clockwise movement in the left velar lobe of a post-hatched veliger.

Figura 4. Captura y transporte de partículas a través del surco alimentario del velo en velígeras de Kelletia kelletii. Las imágenes se aislaron de los videos digitales. Las flechas indican la dirección del movimiento de las partículas. (a, b) Captura de partículas y movimiento en sentido contrario a las agujas del reloj en el lóbulo velar derecho de una velígera post-eclosionada. (c, d) Captura de partículas y movimiento en el sentido de las agujas del reloj en el lóbulo velar izquierdo de una velígera post-eclosionada. 

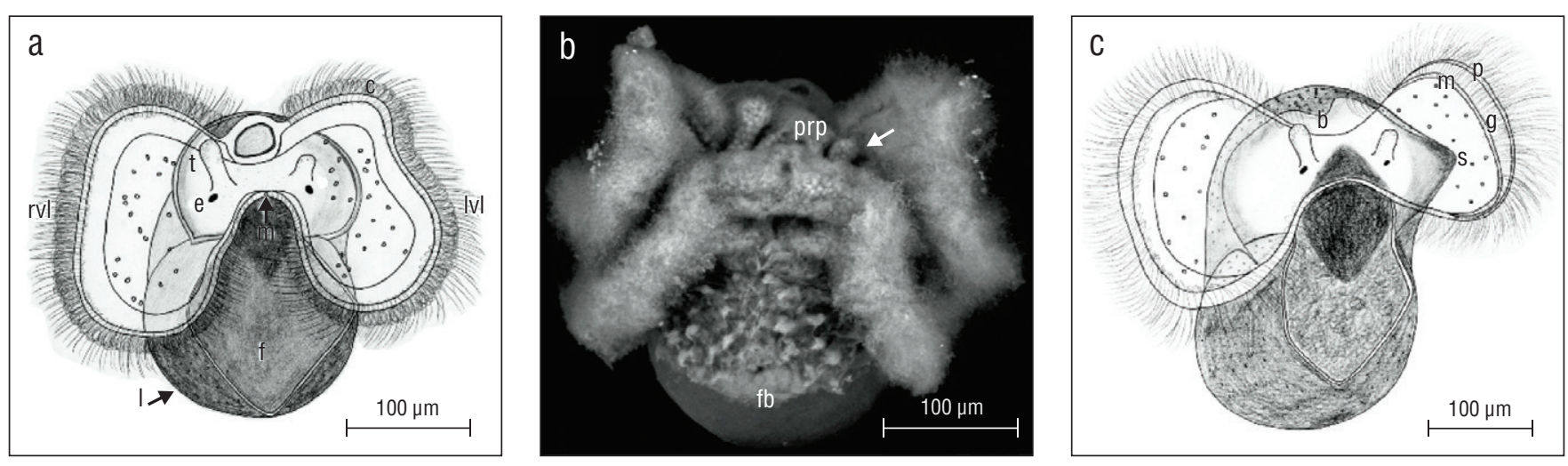

Figure 5. Kelletia kelletii veliger larvae at 2 weeks prior to hatching (a, b) and 2 weeks after hatching (c). (a) Veliger showing symmetrical velar lobes and asymmetrical cephalic tentacles; abbreviations: c, velar cilia; rvl, right velar lobe; lvl, left velar lobe; t, cephalic tentacle; e, eye; m, mouth; f, ciliated foot; 1, larval shell. (b) SEM micrograph of veliger. Arrow indicates smaller left cephalic tentacle; abbreviations: prp, post-oral ciliary patch; fb, foot bristles. (c) Veliger showing asymmetrical velar lobes and asymmetrical cephalic tentacles; abbreviations: b, larval shell beak; g, food grove; s, proto-siphonal canal; $p$, prototroch; m, metatroch.

Figura 5. Larvas velígeras de Kelletia kelletii 2 semanas antes de la eclosión (a, b) y 2 semanas después de la eclosión (c). (a) Velígera que muestra lóbulos velares simétricos y tentáculos cefálicos asimétricos; abreviaturas: c, cilios velares; rvl, lóbulo velar derecho; lvl, lóbulo velar izquierdo; t, tentáculo cefálico; e, ojo; m, boca; f, pie ciliado; 1 , concha larval. (b) Micrografía de velígera mediante microscopía electrónica de barrido. La flecha indica tentáculo cefálico izquierdo más pequeño; abreviaturas: prp, parche ciliar post-oral; fb, cerdas de pie. (c) Velígera que muestra lóbulos velares asimétricos y tentáculos cefálicos asimétricos; abreviaturas: b, pico de concha larvaria; g, surco alimentario; s, canal proto-sifonal; p, prototroca; $m$, metatroca.

veligers fed on micro-particles only after their endogenous yolk stores were depleted; early larval shells were not fully mineralized; and excapsulated veligers had asymmetrical cephalic tentacles and velar lobes.

The substantial range in excapsulation timing in this study, from 39 to $55 \mathrm{~d}$ at $13-16^{\circ} \mathrm{C}$ compared to $30-34 \mathrm{~d}$ at $14.5-17.5^{\circ} \mathrm{C}$ in other studies (Rosenthal 1970, Zacherl et al. 2003), could be due to the cooler water temperatures maintained in this study as well as the minimal water disturbance in parts of the aquarium, which seemed to allow plugging of the capsule escape aperture with masses of undeveloped eggs. In areas of the aquarium that were better aerated and had greater water disturbance, veliger excapsulation was assisted by the water's agitation of the capsules. In still seawater, e.g., in a glass beaker, it was not uncommon for veligers to hatch after $45 \mathrm{~d}$ or more and for many intracapsular veligers to die inside of the capsule.

The asymmetrical development of velar lobes in late-stage $K$. kelletii veligers may counterbalance asymmetrical shell development, which, at that time, includes a proto-siphonal canal. This shell extension is opposite the larger right velar lobe, and when this feature is absent (as in younger veligers), the velar lobes are equal in size. Unequal velar lobes in swimming veligers with asymmetrical shell development has also been observed in other gastropods, including Echinolittorina hawaiiensis (Rosewater \& Kadolsky, 1981) [formerly Littorina picta] (Struhsaker and Costlow 1968). In veligers, right cephalic tentacles that are larger than the left during some stage of larval development have also been observed in other marine snails (e.g., Thais chocolata alimentados por reservas vitelinas endógenas y posiblemente ingirieron albúmina rica en proteínas.

Antes de la eclosión, las reservas vitelinas endógenas de color amarillo brillante ocupaban la mayor parte de la concha larvaria de las velígeras (Fig. 3d). El vitelo se distribuyó en 1 o 2 reservas en forma de esfera que eran visibles a través de la concha translúcida. Si las velígeras eran descapsuladas cuando las reservas vitelinas eran abundantes, estas no ingerían micropartículas. Si se introducían micropartículas a una velígera pre-cría descapsulada, las partículas de pigmento quedaban atrapadas en los lóbulos velares, se asentaban en el surco alimentario y se movían hacia la boca donde eran expulsadas por el pie ciliado. Las velígeras no ingirieron partículas a menos que sus reservas vitelinas se agotaran (e.g., en algunos casos a los $18 \mathrm{~d}$ en el plancton). La ingestión de micropartículas fue confirmada por el color brillante de las micropartículas DayGlo visibles dentro del intestino.

\section{Morfología de la concha larvaria}

Las conchas de las velígeras intracapsulares tardías pre-eclosionadas eran delgadas, quebradizas y, a menudo, tenían agujeros que indicaban una pobre mineralización de la concha (Fig. 3f). Las conchas de las velígeras emergentes eran similares a las de las pre-crías, pero estaban un poco más mineralizadas y no eran tan quebradizas. Entre los días 13 y 15 como velígeras, las conchas larvarias estaban completamente mineralizadas y las líneas de crecimiento eran evidentes (Fig. 6a, b, c). A los 20 d, o cerca de los 20 d, las velígeras en etapa tardía eran velígeras nadadoras descapsuladas que 

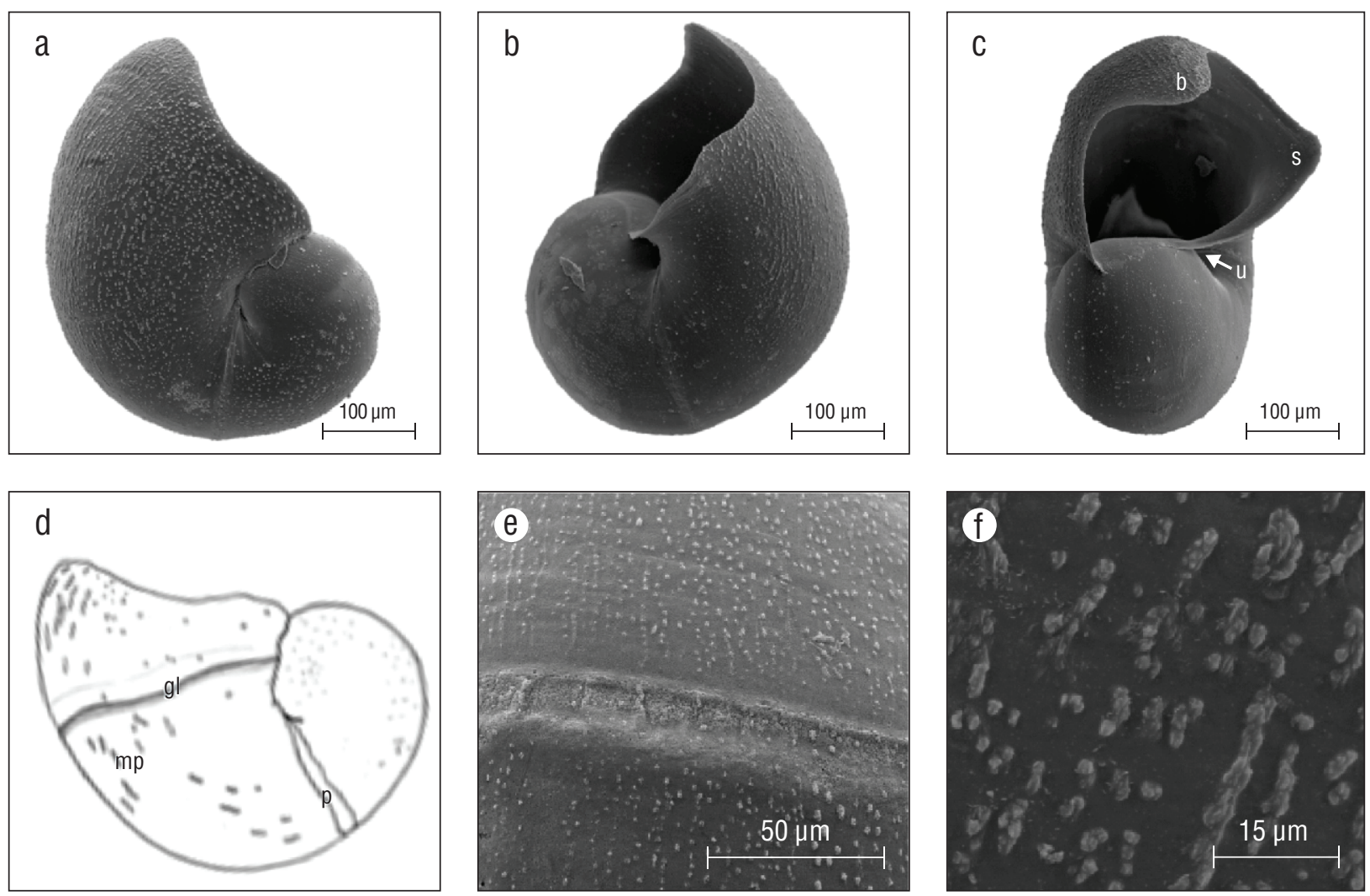

Figure 6. Kelletia kelletii larval shells. (a-c) SEM micrographs of larval shells of veliger larvae 2 weeks after hatching, (d) line drawing of late-stage veliger shells with labeled features, (e) SEM micrograph of protoconch 1 boundary, and (f) SEM micrograph of larval shell micro-protuberances. Abbreviations: b, apertural beak; u, umbilicus; s, proto-siphonal canal; gl, shell boundary indicating shell size at hatching; mp, bead-like micro-protuberances; p: boundary/break in growth of protoconch 1.

Figura 6. Conchas larvarias de Kelletia kelletii. (a-c) Micrografías de la microscopía electrónica de barrido (MEB) de conchas larvarias de larvas velígeras 2 semanas después de la eclosión, (d) dibujo lineal de conchas velígeras en etapa tardía con etiquetas de las características, (e) micrografía MEB del límite de la protoconcha 1 y (f) micrografía MEB de micro-protuberancias de la concha larvaria. Abreviaturas: b, pico de apertura; u, ombligo; s, canal proto-sifonal; gl, límite de la concha que indica el tamaño de la concha al momento de la eclosión; mp, microprotuberancias en forma redondeada; p: límite/ruptura en el crecimiento de la protoconcha 1.

(Duclos, 1832) (Romero et al. 2004) and Amphissa versicolor Dall, 1871 (Page and Parries 2000).

Dense taxon sampling is necessary within the Buccinidae, and other gastropod groups, for phylogenetic analyses that can elucidate the evolution and distribution of larval developmental mode (Collin and Moran 2018). Of course, such studies are only as good as the larval data examined with the phylogeny. Therefore, detailed data for this species, and others, of larval developmental timing, morphology, nutritive eggs, and intracapsular and extracapsular feeding mode are essential to inferring homology, understanding ancestral versus derived character states (Collin and Moran 2018), and making novel insights about evolutionary patterns in larval evolution.

\section{ACKNOWLEDGMENTS}

Funding from the UC Berkeley Department of Integrative Biology and Sigma Xi made this study possible. The author tenían un pico de apertura en la concha larvaria y el comienzo de un canal sifonal (canal proto-sifonal) (Fig. 5c, 6c). En las conchas de las velígeras de diferentes edades, se evidenciaron microprotuberancias con forma redondeada hasta el margen de apertura (Fig. 6d, e, f). El diámetro máximo de las conchas larvarias de K. kelletii fue $0.407 \mathrm{~mm}(n=10)$ en vista apical. Sin embargo, el tamaño general varió entre los individuos dentro de las cápsulas y las cohortes no siempre presentaron la misma etapa de desarrollo y calcificación de la concha, especialmente las velígeras pre-crías y las emergidas en estadios tempranos.

\section{Discusión}

Muchas observaciones de $K$. kelletii reportadas aquí son consistentes con las documentadas en otros estudios (e.g., por MacGinitie y MacGinitie 1949, Rosenthal 1970, Morris et al. 1980, Zacherl et al. 2003, Zacherl 2005, Romero et al. 2012, Rodríguez 2017, Wilson 2017). Por ejemplo, las larvas 
is grateful to Ray Thompson (Memorial University, Canada) and Oscar Chaparro (Universidad Austral de Chile, Chile) for referring me to the DayGlo Company of Ohio who produced the micro-particles used in larval feeding experiments. For larval relaxation techniques, the advice and protocols of Louise Page (University of Victoria, Canada) were invaluable, as were conversations with Janice Voltzow (University of Scranton, PA) and Carole Hickman (UC Berkeley). Thanks as well to Kent McDonald, Guangwei Min, and Reena Zalpuri at The Robert D. Ogg Electron Microscope Laboratory at UC Berkeley for assistance with larval specimen manipulation, critical point drying, and SEM imaging. Tim Herrlinger (UC Berkeley) and Shane Anderson (UC Santa Barbara) collected all specimens, for which I am sincerely grateful. The careful edits and suggestions of 2 anonymous reviewers greatly improved this paper.

\section{REFERENCES}

Buckland-Nicks J, Gibson G, Koss R. 2002. Phylum Mollusca: Gastropoda. In: Young CM, Sewell MA, Rice ME (eds.), Atlas of Marine Invertebrate Larvae. New York (USA): Academic Press. 640 p.

Collin R, Moran A. 2018. Evolutionary transitions in mode of development. In: Carrier TJ, Reitzel AM, Heyland A (eds.), Evolutionary Ecology of Marine Invertebrate Larvae. UK: Oxford University Press. p. 50-66. https://doi.org/10.1093/oso/9780198786962.003.0004

Herrlinger TJ. 1981. Range extension of Kelletia kelletii. Veliger. 24(1):78.

Horwitz R, Jackson MD, Mills SC. 2017. The embryonic life history of the tropical sea hare Stylocheilus striatus (Gastropoda: Opisthobranchia) under ambient and elevated ocean temperatures. PeerJ. 5:e2956. https://doi.org/10.7717/peerj.2956

[Image J] Image Processing and Analysis in Java. 2004. Image J. Bethesda (Maryland, USA): National Institutes of Health; accessed 1997-2018. http://rsb.info.nih.gov/ij/

Lonhart SI. 2001. An invasive whelk as predator and prey: the ecology of Kelletia Kelletii in Monterey Bay, California [dissertation]. [Santa Cruz (California)]: University of California. $228 \mathrm{p}$.

MacGinitie GE, MacGinitie N. 1949. Natural History of Marine Animals 2nd ed. New York: McGraw-Hill. 512 p.

McLean JH. 1978. Marine Shells of Southern California. 2nd ed. Science Series 24(11). Los Angeles (California): Natural History Museum of Los Angeles County. 104 p.

Miloslavich P, Penchaszadeh PE. 1994. Spawn and development of Engoniophos unicinctus (Say, 1825) (Gastropoda: Prosobranchia) from the southern Caribbean Sea. Veliger. 37(4):425-429.

Morris RH, Abbott DP, Haderlie EC. 1980. Intertidal Invertebrates of California. California: Stanford Press. 928 p.

Page LR, Parries SC. 2000. Comparative study of the apical ganglion in planktotrophic caenogastropod larvae: ultrastructure and immunoreactivity to serotonin. J Comp Neurol. 418(4):383-401.

h t t p s : //d o i.org / $10.1002 /$ ( s i c i ) 1096 9861(20000320)418:4<383::aid-cne2>3.0.co;2-b

Pearce JB, Thorson G. 1967. The feeding and reproductive biology of the red whelk, Neptunea antiqua (L.) (Gastropoda, Prosobranchia). Ophelia. 4(2):277-314.

https://doi.org/10.1080/00785326.1967.10409624 no se alimentan dentro de sus ovicápsulas, las larvas descapsuladas nadan y se alimentan en el plancton como velígeras y las larvas desxcapsuladas son fototáxicas. Cabe destacar que aquí se informan por primera vez varias observaciones de la morfología y el desarrollo de las larvas: todas las ovicápsulas examinadas incluían huevos con un desarrollo detenido o no fertilizados; después de emerger de sus cápsulas, las velígeras se alimentaron de micropartículas solo cuando habían agotado sus reservas vitelinas endógenas; las conchas larvarias tempranas no estaban completamente mineralizadas; y las velígeras descapsuladas tenían tentáculos cefálicos y lóbulos velares asimétricos.

El extenso intervalo en el tiempo de descapsulación observado en este estudio, de 39 a $55 \mathrm{~d}$ con una temperatura de $13-16^{\circ} \mathrm{C}$ en comparación con los 30-34 d con una temperatura de a $14.5-17.5^{\circ} \mathrm{C}$ observado en otros estudios (Rosenthal 1970, Zacherl et al. 2003), podría deberse a las temperaturas más frías que se mantuvieron en este estudio y a la mínima perturbación del agua en algunas partes del acuario, lo que pareció permitir la obstrucción de la apertura de escape de la cápsula con masas de huevos no desarrollados. En las áreas del acuario que estaban mejor aireadas y tenían una mayor perturbación del agua, la descapsulación de las velígeras fue asistida por la agitación de las cápsulas ocasionada por el agua. En agua sin movimiento, e.g., en un vaso de precipitado de vidrio, fue común que las velígeras eclosionaran después de $45 \mathrm{~d}$ o más y que muchas velígeras intracapsulares murieran dentro de la cápsula.

El desarrollo asimétrico de los lóbulos velares en las velígeras de $K$. kelletii en etapa tardía puede contrarrestar el desarrollo asimétrico de la concha, el cual, en ese momento, incluye un canal proto-sifonal. Esta extensión de la concha se encuentra opuesta al lóbulo velar derecho, que es de mayor tamaño, y cuando esta característica está ausente (como en las velígeras más jóvenes), los lóbulos velares presentan el mismo tamaño. También se han observado lóbulos velares desiguales en velígeras nadadoras con un desarrollo asimétrico de la concha en otros gasterópodos, como Echinolittorina hawaiiensis (Rosewater \& Kadolsky, 1981) [antes Littorina picta] (Struhsaker y Costlow 1968). En velígeras, también se han observado tentáculos cefálicos que son más grandes en el lado derecho que en el izquierdo durante alguna etapa del desarrollo larvario en otros caracoles marinos (e.g., Thais chocolata (Duclos, 1832) (Romero et al. 2004) y Amphissa versicolor (Dall, 1871) (Page y Parries 2000).

Se requiere un muestreo de taxones denso dentro de los Buccinidae, y otros grupos de gasterópodos, para los análisis filogenéticos que puedan dilucidar la evolución y la distribución del modo de desarrollo larvario (Collin y Moran 2018). Por supuesto, estos estudios solo son tan buenos como los datos larvarios examinados con la filogenia. Por lo tanto, los datos detallados para esta especie, y otras, sobre el tiempo de desarrollo de las larvas, la morfología, los huevos nutritivos y el modo de alimentación intracapsular y extracapsular son 
Pechenik JA. 1979. Role of encapsulation in invertebrate life histories. Am Nat. 114(6):859-870. https://doi.org/10.1086/283533

Pedersen RVK, Page LR. 2000. Development and metamorphosis of the planktotrophic larvae of the moon snail, Polinices lewisii (Gould, 1847) (Caenogastropoda: Naticoidea). Veliger. 43(1):58-63.

Power AJ, Keegan BF. 2001. The significance of imposex levels and TBT contamination in the red whelk, Neptunea antiqua (L.) from the offshore Irish Sea. Mar Pollut Bull. 42(9):761-772. https://doi.org/10.1016/s0025-326x(00)00221-6

Rodriguez KN. 2017. Linking large scale ocean-atmospheric patterns with recruitment in Kellet's whelk (Kelletia kelletii). [Undergraduate Senior Project]. [San Luis Obispo (California)]: Biological Sciences Department California Polytechnic State University. $19 \mathrm{p}$.

Romero MS, Gallardo CS, Bellolio G. 2004. Egg laying and embryonic-larval development in the snail Thais (Stramonita) chocolata (Duclos, 1832) with observations on its evolutionary relationships within the Muricidae. Mar Biol. 145:681-692. https://doi.org/10.1007/s00227-004-1368-9

Romero MR, Walker KM, Cortez CJ, Sanchez Y, Nelson KJ, Ortega DC, Smick SL, Hoese WJ, Zacherl DC. 2012. Larval diel vertical migration of the marine gastropod Kelletia kelletii (Forbes, 1850). J Mar Biol. 2012:386575. https://doi.org/10.1155/2012/386575

Rosenthal RJ. 1970. Observations on the reproductive biology of the Kellet's whelk, Kelletia kelletii (Gastropoda Neptunidae). Veliger. 12(3):319-324.

Struhsaker JW, Costlow JD Jr. 1968. Larval development of Littorina picta (Prosobranchia, Mesogastropoda), reared in the laboratory. J Molluscan Stud. 38(2):153-160. https://doi.org/10.1093/oxfordjournals.mollus.a065033

Tan KS, Morton B. 1998. The ecology of Engina armillata (Gastropoda: Buccinidae) in the Cape d'Aguilar Marine Reserve, Hong Kong, with particular reference to its preferred prey (Polychaeta: Serpulidae). J Zool. 244(3):391-403. https://doi.org/10.1111/j.1469-7998.1998.tb00044.x

Thorson G. 1950. Reproductive and larval ecology of marine bottom invertebrates. Biol Rev. 25(1):1-45. https://doi.org/10.1111/j.1469-185x.1950.tb00585.x

Wilson M. 2017. Understanding the transition from dispersive larva to benthic adult: A study of the environmental factors that impact Kelletia kelletii larval settlement. [Undergraduate Senior Project]. [San Luis Obispo (California)]: Biological Sciences Department California Polytechnic State University. esenciales para inferir la homología, comprender los estados de carácter ancestrales contra los derivados (Collin y Moran 2018) y generar nuevos conocimientos sobre patrones evolutivos en la evolución larvaria.

\section{Agradecimientos}

Los fondos del Departamento de Biología Integrativa de UC Berkeley y Sigma Xi hicieron posible este estudio. El autor agradece a Ray Thompson (Memorial University, Canadá) y Oscar Chaparro (Universidad Austral de Chile, Chile) por recomendar la empresa DayGlo de Ohio, que produjo las micropartículas utilizadas en los experimentos de alimentación de larvas. Para las técnicas de relajación larvaria, los consejos y protocolos de Louise Page (Universidad de Victoria, Canadá) fueron invaluables, al igual que las conversaciones con Janice Voltzow (Universidad de Scranton, PA) y Carole Hickman (UC Berkeley). Gracias también a Kent McDonald, Guangwei Min y Reena Zalpuri del Laboratorio de Microscopios Electrónicos Robert D. Ogg en UC Berkeley por su ayuda con la manipulación de las muestras larvales, el secado de puntos críticos y las imágenes MEB. Tim Herrlinger (UC Berkeley) y Shane Anderson (UC Santa Barbara) recolectaron todos los especímenes, por lo cual estoy sinceramente agradecida. Las cuidadosas ediciones y sugerencias de 2 revisores anónimos mejoraron enormemente este documento.

Traducido al español por Claudia Michel-Villalobos.

Zacherl DC. 2005. Spatial and temporal variation in statolith and protoconch trace elements as natural tags to track larval dispersal. Mar Ecol Prog Ser. 290:145-163. https://doi.org/10.3354/meps290145

Zacherl D, Gaines SD, Lonhart SI. 2003. The limits to biogeographical distributions: insights from the northward range extension of the marine snail, Kelletia kelletii (Forbes, 1852). J Biogeogr. 30(6):913-924. https://doi.org/10.1046/j.1365-2699.2003.00899.x

Received February 2020, accepted August 2020. 